\title{
Hsa_circ_0001461 as a novel biomarker in patients with primary focal segmental glomerulosclerosis
}

\section{Type}

Research paper

\section{Keywords}

nephrotic syndrome, minimal change disease, Circular RNAs, focal segmental glomerulosclerosis

\begin{abstract}
Introduction

Circular RNAs (circRNAs), a novel class of non-coding RNAs, have been implicated in various diseases and are considered to play an important role in physiological and pathological processes. We aimed to profile renal circRNA in children with primary focal segmental glomerulosclerosis (FSGS) and predict the potential mechanism underlying the pathogenesis of FSGS.
\end{abstract}

Material and methods

A global circRNA expression analysis was performed in renal tissues from patients with FSGS $(n=3)$ and controls $(n=3)$. Quantitative real-time polymerase chain reaction (qRT-PCR) was conducted to validate the expression levels of three circRNAs in a cohort of 44 patients with FSGS, 44 agematched healthy controls, and 15 patients with minimal change disease (MCD).

\section{Results}

A total of 152 circRNAs were differentially expressed in patients with FSGS compared with controls. Hsa_circ_0001461 and hsa_circ_0000605 were significantly increased in patients with FSGS compared with controls, as determined by qRT-PCR $(p<0.001)$. The receiver operating characteristic (ROC) curve areas of hsa_circ_0001461 and hsa_circ_0000605 were 0.948 and 0.934 , respectively. Hsa_circ_0001461 was significantly elevated in patients with FSGS compared with those with MCD and was correlated with $24 \mathrm{~h}$ urinary protein in patients with FSGS $(r=0.732, p<$ 0.001 ). Bioinformatics analysis predicted mir-30a interaction with hsa_circ_0001461. Mir-30a was negatively correlated with the renal hsa_circ_0001461 level $(r=-0.90, p<0.001)$.

\section{Conclusions}

Hsa_circ_0001461 is identified as a novel biomarker in children with FSGS and might provide insights into the mechanism of FSGS pathogenesis. 
Hsa_circ_0001461 as a novel biomarker in patients with primary focal segmental glomerulosclerosis

\section{Abstract}

Introduction: Circular RNAs (circRNAs), a novel class of non-coding RNAs, have been implicated in various diseases and are considered to play an important role in physiological and pathological processes. We aimed to profile renal circRNA in children with primary focal segmental glomerulosclerosis (FSGS) and predict the potential mechanism underlying the pathogenesis of FSGS.

Material and methods: A global circRNA expression analysis was performed in renal tissues from patients with FSGS $(n=3)$ and controls $(n=3)$. Quantitative real-time polymerase chain reaction (qRT-PCR) was conducted to validate the expression levels of three circRNAs in a cohort of 44 patients with FSGS, 44 age-matched healthy controls, and 15 patients with minimal change disease (MCD).

Results: A total of 152 circRNAs were differentially expressed in patients with FSGS compared with controls. Hsa_circ_0001461 and hsa_circ_0000605 were significantly increased in patients with FSGS compared with controls, as determined by qRT-PCR $(p<0.001)$. The receiver operating characteristic (ROC) curve areas of hsa_circ_0001461 and hsa_circ_0000605 were 0.948 and 0.934 , respectively. Hsa_circ_0001461 was significantly elevated in patients with FSGS compared with those with MCD and was correlated with $24 \mathrm{~h}$ urinary protein in patients with FSGS ( $\mathrm{r}$ $=0.732, \mathrm{p}<0.001)$. Bioinformatics analysis predicted mir-30a interaction with hsa_circ_0001461. Mir-30a was negatively correlated with the renal 
hsa_circ_0001461 level $(r=-0.90, p<0.001)$.

Conclusions: Hsa_circ_0001461 is identified as a novel biomarker in children with FSGS and might provide insights into the mechanism of FSGS pathogenesis.

Key words circular RNAs, focal segmental glomerulosclerosis, minimal change disease, nephrotic syndrome

\section{Introduction}

Primary focal segmental glomerulosclerosis (FSGS), resulting from podocyte injury, is a clinicopathological entity that manifests as massive proteinuria, edema, and hypoalbuminemia, accounting for $10-20 \%$ of idiopathic nephrotic syndrome (NS) in children [1]. FSGS often shows steroid resistance, therapeutic dilemma, and a tendency to progress to end-stage renal disease (ESRD) [2]. Due to the profound renal morbidity of FSGS, it has become the subject of intensive basic scientific and clinical research over the past sixty years. To our knowledge, the precise pathogenesis of FSGS remains unclear.

FSGS and MCD have a similar clinical presentation and diverse prognosis. The relationship between MCD and primary FSGS remains controversial. The diagnosis of these two entities is a challenge for the nephropathologists owing to the bias of sampling under some circumstances. CD80 [3], uPAR [4] and miRNAs [5] have been reported as biomarkers to differentiate FSGS from MCD. the research based on different circRNAs between FSGS and MCD is still blank.

Circular RNAs (circRNAs) are a large class of non-coding RNAs that are produced 
by backsplicing [6]. Because of the absence of the 5' cap and 3' end, circRNAs are resistant to exonuclease and stable in tissues [7-8]. circRNAs show tissue-specific and cell-specific expression patterns [9-10] and are implicated in multiple diseases such as cancers [11], cardiovascular disorders [12], and diabetes mellitus [13]. They regulate gene expression in various ways, including acting as miRNA sponges [7, 14]. RNA binding protein sponges [15] and protein templates [16-18]. Recently, hsa_circ_007500 (circHLA-C) was found to be significantly elevated in kidney tissues from patients with lupus nephritis $(\mathrm{LN})$ and was proposed to play a vital role in the pathogenesis of LN by sponging mir-150 [19]. The profiling of circRNAs in peripheral blood has been reported in acute kidney injury and membranous nephropathy. circLRP6 could regulate mesangial cell proliferation, oxidative stress, extracellular matrix accumulation, and inflammation by sponging miR-205, upregulating HMGB1, and activating the TLR4/NF- $\kappa$ B pathway in diabetic nephropathy [20]. circRNA_15698 was significantly upregulated in diabetic mice and promoted the synthesis of extracellular matrix-related proteins through miR-185/TGF- $\beta 1$ [21]. To date, the circRNA expression profile of primary FSGS in children remains unknown.

microRNAs (miRNAs), endogenous non-coding RNAs, have been intensively explored in the pathogenesis of renal diseases. Mir-30a is essential for the development of the kidney [22]. Serum mir-30a was upregulated in children with nephrotic syndrome and correlated with proteinuria [23]. The level of serum mir-30a was associated with the activity of FSGS [24]. Multiple studies indicated that mir-30a plays a vital role in the process of podocyte injury and renal fibrosis [25]. However, the 
regulatory mechanism between circRNAs and miRNAs has not yet been elucidated.

In the present study, we first investigated the expression profile of circRNAs in renal tissues from individuals with primary FSGS by high-throughput sequencing. Second, we compared candidate circRNAs in the whole cohort of our study by quantitative reverse transcription-polymerase chain reaction (qRT-PCR). Finally, we conducted a bioinformatics analysis to predict the competing endogenous RNA (ceRNA) of candidate circRNAs.

\section{Materials and methods}

Patients and specimens

A total of 109 participants were enrolled in the current study from the Guangzhou Women and Children's Medical Center between July 2018 and January 2020. Patients with FSGS ( $\mathrm{n}=47,3$ for genome-wide expression analysis, 44 for circRNAs validation) and MCD ( $n=15)$ were consecutively recruited from the Department of Nephrology of the Guangzhou Women and Children's Medical Center. Renal biopsies were performed in children with NS, who had initial or secondary steroid resistance, reduced serum complement, or impaired renal function. The inclusion criteria for FSGS/MCD were as follows: age between 1 and $18 \mathrm{y}$; diagnosed with NS according to the ISKDC criteria; patients in recurrence at the time of kidney biopsy; and renal pathological changes confirmed by an expert. Renal pathological diagnosis was made by light microscopy (LM), immunofluorescence and transmission electron microscopy (TEM). FSGS was diagnosed morphologically by the presence of focal and segmental 
sclerosis by LM with foot process effacement as seen by TEM. MCD was diagnosed by the presence of morphological features of foot process effacement in TEM, while no glomerular lesions were captured by LM. Both FSGS and MCD showed negative or low-intensity mesangial IgM (sometimes accompanied by C3 or C1q) staining detected in immunofluorescence. The exclusion criteria for FSGS/MCD were as follows: genetic mutations contributing to steroid-resistant nephrotic syndrome, lupus nephritis, IgA nephropathy, Henoch-Schonlein purpura nephritis, hepatitis B-related nephritis, hepatitis C-related nephritis, and other secondary nephritis, and estimated glomerular filtration rate (eGFR) lower than $30 \mathrm{ml} / \mathrm{min} / 1.73 \mathrm{~m}^{2}$ at least lasting for three months. In addition, 47 children ( 3 for genome-wide expression analysis, 44 for circRNAs validation) with renal mass (histologically confirmed as nephroblastoma or clear cell carcinoma), regarded as controls, were enrolled from the surgical oncology department of the Guangzhou Women and Children's Medical Center. Renal tissues as controls were obtained from renal parenchyma at least $5 \mathrm{~cm}$ from the edge of the renal mass. Percutaneous renopuncture biopsy was performed to obtain three or four pieces of renal tissues $(1.0-1.5 \mathrm{~cm}$ in length) from each patient with FSGS/MCD. One piece of renal tissue was used for histologic diagnosis, the remaining pieces were all used for research. The design strategy is demonstrated in Figure 1. The present study was approved by the Institutional Review Board of the Guangzhou Women and Children's Medical Center. An estimated total sample size of 54 (allocation ratio $=1$ ) would be needed to provide $80.41 \%$ Power to reject the null hypothesis of equal means with a significance level of 0.05 using a two-sample Wilcoxon-Mann-Whitney test by G 
Power. The written informed consents were obtained from legal representatives of all participants before the percutaneous renal biopsy or surgery in Guangzhou Women and Children's Medical Center.

Profiling

Total RNA was extracted from kidney tissues from patients with FSGS $(n=3)$ and controls ( $n=3)$ using TRIzol Reagent (Invitrogen) according to the manufacturer's protocols. The concentration of the RNA was qualified and quantified using NanoDropND1000. The integrity of the RNA was analyzed by $1 \%$ agarose gel electrophoresis. Isolated RNA was stored at $-80^{\circ} \mathrm{C}$ until further analysis.

RNA library construction and circRNA sequencing were performed by Ribo Biotech Inc. (Guangzhou, China). A Ribo-Zero rRNA removal kit and Rnase-R were used to remove rRNA and linear RNA from the total RNA, respectively. Then, the rRNAdepleted RNA was processed with a TruSeq Stranded RNA Library Prep Kit (Illumina, USA) to establish the RNA library. The library was qualified and quantified using the BioAnalyzer 2100 system (Agilent Technologies, USA). The RNA libraries were denatured as single-stranded DNA, captured on Illumin Flow cells, amplified in situ as clusters, and then sequenced on an Illumina Hi-Seq sequencing instrument (PE 150 mode). The effective reads were eligible for circRNA analysis following 3 adapter trimming and the removal of poor-quality reads.

Hierarchical clustering analysis was performed to distinguish the expression levels of circRNAs between the FSGS and controls. CircRNAs with fold changes $>2.0$ and $p$-values $<0.05$ were defined as significant differential expressions. 
Validation of candidate circRNAs and miRNAs

The total RNA of kidney tissues was reverse transcribed into cDNA with dNTP Mix (HyTest Ltd), RNase Inhibitor (Epicenter, Illmina, Inc.), and SuperScript III (Thermo Fisher Scientific, Inc.). Due to the low abundance of circRNAs and limited renal tissue through renopuncture biopsy, circRNAs with high expression levels were selected as candidate circRNAs. Candidate circRNAs were validated by real-time polymerase chain reaction (PCR) on an ABIViiA 7 Real-time PCR System (Applied Biosystems, Foster City, CA, USA). The divergent junction-spanning primers for specific detection of circRNAs are listed in Table I . $\beta$-actin was used as an endogenous control gene for circRNAs. The mir-30a expression level in the kidney was also assessed using a TaqMan PCR kit (Applied Biosystems, Foster City, CA, USA) on an ABIViiA 7 Realtime PCR System (Applied Biosystems, Foster City, CA, USA), and let-7 was chosen as an internal control. Relative expression was analyzed by the $\Delta \Delta \mathrm{Ct}$ analysis.

Statistical analysis

Statistical analysis was performed using SPSS 18.0 (Chicago, IL, USA) and GraphPad Prism 5.0 (GraphPad Software, CA, USA). Baseline statistics were represented as the mean $\pm S D$ or median $\left(P_{25}, P_{75}\right)$ for quantitative data and numbers for categorical data. The Mann-Whitney $U$ test or Student's t-test was used to compare differences in circRNA expression levels between groups. The chi-square test or Fisher's exact test was performed between two categorical variables. Receiver operating characteristic (ROC) curves were obtained, and the specificity and sensitivity of predictive power were assessed by area under the curve (AUC). Correlations 
between circRNAs and clinical parameters or miRNA levels were analyzed using Pearson's correlation analysis. Statistical significance was determined at $p<0.05$.

\section{Results}

CircRNA expression profile in renal tissues

To analyze the profiling of circRNAs in FSGS, we conducted RNA-deep sequencing of RNA extracted from the kidney tissues of three patients with FSGS and three normal kidney tissues. The results revealed that a total of 6322 circRNAs were detected in the kidney, of which 152 circRNAs were differentially expressed in patients with FSGS compared with controls (fold changes $\geq 2.0, p$-values $<0.05$ ). Among these abnormally expressed circRNAs, 79 circRNAs were upregulated, and 73 were downregulated, as shown on the cluster heatmap and volcano plots (Figure 2A and B). The top ten differentially expressed circRNAs between FSGS patients and controls are shown in Table II.

Validation of circRNAs by qRT-PCR

We selected the candidate circRNAs with high expression levels in both groups to ensure stable detection as well as remarkably differential expression. Subsequently, hsa_circ_0001461, hsa_circ_0000605, and hsa_circ_0001849 were verified in kidney tissues from a subset of FSGS patients $(n=30)$ and controls $(n=30)$ by qRT-PCR. The demographic and baseline characteristics of FSGS patients and controls are summarized in Table III. The results indicated that hsa_circ_0001461 and hsa_circ_0000605 were both significantly increased in patients with FSGS $(p<0.001$, 
$p<0.001$, respectively) (Figure $3 A$ and $B$ ); however, no significant difference in the hsa_circ_0001849 level was found between the two groups ( $p>0.05)$ (Figure 3C).

ROC curves were obtained to validate the potential diagnostic value of hsa_circ_0001461 and hsa_circ_0000605 for FSGS. The ROC curves of candidate circRNAs indicated that hsa_circ_0001461 (AUC 0.948, 95\% Cl 0.898-0.997, p < 0.001) and hsa_circ_0000605 (AUC 0.934, 95\% Cl 0.876-0.993, p < 0.001) were capable of differentiating patients with FSGS from controls (Figure 4A and B).

Correlation of circRNA fold changes with laboratory parameters in FSGS

Subsequently, we analyzed the correlation between the expression fold changes of hsa_circ_0001461/hsa_circ_0000605 and laboratory parameters (24 h urinary protein, serum creatinine, eGFR) in the FSGS group. We found that hsa_circ_0001461 was positively correlated with $24 \mathrm{~h}$ urinary protein in patients with FSGS $(r=0.732, p$ $<0.001$ ) (Figure 4C). However, there was no significant relationship between hsa_circ_0001461 and other laboratory indexes, and hsa_circ_0000605 was not correlated with laboratory parameters in patients with FSGS.

Comparison of circRNAs among FSGS, MCD, and controls

We next compared the concentrations of hsa_circ_0001461 and hsa_circ_0000605 in a cohort of patients with FSGS $(n=14)$, patients with MCD $(n=$ 15), and age-matched controls $(n=14)$. The demographic and baseline characteristics of the patients are summarized in Table IV. No significant differences were observed in age, sex, serum albumin levels, $24 \mathrm{~h}$ urine protein, and eGFR between the FSGS and MCD groups. Hsa_circ_0001461 was expressed much higher in kidney samples 
from patients with FSGS than in those with MCD and controls $(p<0.001)$ (Figure 5A). No significant difference was found between patients with MCD and controls $(p>0.05)$. In addition, patients with FSGS had higher concentrations of hsa_circ_0000605 than controls $(p<0.001)$ (Figure 5B). However, there was no significant difference between patients with FSGS and MCD ( $p$ > 0.05). This suggests that hsa_circ_0001461 might be a potential novel biomarker to distinguish FSGS from MCD.

Prediction of the ceRNA for hsa_circ_0001461

Hsa_circ_0001461, an extronic circular RNA, contains several binding sites for AGO2, which is essential for the microRNA sponge function. Renal hsa_circ_0001461 had the greatest fold change in patients with FSGS compared with those with MCD and controls. Renal hsa_circ_0001461 was significantly associated with the level of proteinuria in FSGS. Mir-30a was predicted to interact with hsa_circ_0001461 by TargetScan and miRanda (Figure 6A). Hsa_circ_0001461 contained eight binding sites for mir-30a. We found that mir-30a was negatively correlated with the renal level of hsa_circ_0001461 $(r=-0.90, p<0.001)$ (Figure 6B).

\section{Discussion}

In this study, we identified 152 aberrantly expressed circRNAs in kidney tissues by next-generation sequencing and then validated three circRNAs (hsa_circ_0001461, hsa_circ_0000605, and hsa_circ_0001849) with high transcript levels and obvious differences in patients with FSGS and controls in a larger cohort. Hsa_circ_0001461 and hsa_circ_0000605 were confirmed to be significantly upregulated in patients with 
FSGS by qRT-PCR. Patients with FSGS had higher levels of hsa_circ_0001461 compared with patients with MCD and controls. In addition, hsa_circ_0001461 was positively associated with proteinuria in FSGS. Hence, we considered hsa_circ_0001461 as a potential novel biomarker for FSGS. The mir-30a was predicted to be a potential binding target of hsa_circ_0001461, and mir-30a displayed a negative correlation with hsa_circ_0001461, which indicated that hsa_circ_0001461 might function as a miRNA sponge to participate in the pathogenesis of FSGS.

Almost $90 \%$ of the human genome is transcribed into non-coding RNA transcripts, of which long non-coding RNAs have been extensively studied. Thousands of circRNAs have been discovered in eukaryotes with the rapid development of highthroughput RNA sequencing $[8,26]$. In recent decades, many studies concerned with circRNAs have been found that might serve as potential biomarkers due to their stability and tissue-specific expression pattern [9-10]. HHLA2 and circ_ABCB10 were shown to be significantly increased in renal cell carcinoma and correlated with short overall survival [27-28]. A study found that serum cirR_126 was upregulated in patients with acute kidney injury (AKI) and considered it as a probable biomarker and predictor of mortality in AKI [29]. In another study, circ_101319 in the peripheral blood was significantly higher and exhibited promising diagnostic value in patients with idiopathic membranous nephropathy (IMN) [30]. MUC3A in exosomes from serum and urine was considered a potential biomarker for IMN [31]. Plasma circRNA_002453 was shown to be a novel biomarker in the diagnosis of lupus nephritis [32]. In the current study, hsa_circ_0001461 and hsa_circ_0000605 were significantly increased in patients with 
FSGS compared to controls. ROC curve analysis showed that hsa_circ_0001461 and hsa_circ_0000605 could distinguish patients with FSGS from controls.

There are controversies regarding whether FSGS and MCD are the same disease or distinct entities. Some researchers believe that although both are classified as podocytopathy, they are supposed to have different development mechanisms [33]. Differentiation between FSGS and MCD depends on the renal biopsy and the experience of the nephropathologist. The search for reliable biomarkers to distinguish FSGS from MCD is still underway. Previous studies have shown that serum mir-192, mir-205 [5] and urinary/renal uPAR [4, 34] have the potential to differentiate FSGS from MCD. In our study, hsa_circ_0001461 was expressed at higher levels in patients with FSGS than in those with MCD and controls.

Hsa_circ_0001461 has been reported in a small number of studies related to the tumor. Hsa_circ_0001461 was found upregulated and prompted osteosarcoma progression and metastasis by sponging mir-181b [35]. Hsa_circ_0001461 participated in the progression of osteosarcoma by sponging miR-375 to upregulate the expression of Yes-associated protein 1 [36]. In addition, hsa_circ_0001461 prompted the tumor proliferation and migration via inhibiting the mir-873 expression and increasing the ZEB1 expression in papillary thyroid cancer [37]. On the other hand, circ_0001461 was decreased in gastric cancer and inhibited the tumor progression through regulating RUNX1 and YBP-1 [38]. The role of circ_0001461 in nephropathy needs intensive exploration in the future.

Hsa_circ_0001461, an extronic circular RNA, contains several binding sites for 
AGO2, which is essential for microRNA sponge function. The mir-30a was predicted as potential targets of hsa_circ_0001461 through bioinformatics analysis. Mir-30a were verified to be abundant in podocytes and tubular epithelial cells and play a vital role in the physiological and pathological processes in the kidney [39-40]. Mir-30a regulates the function of podocytes through calcium/calcineurin signaling by targeting TRPC6, PPP3CB, and NFATC3 [41]. In addition, mir-30a inhibits apoptosis in podocytes by targeting Notch1 and P53 through Notch signaling and p53 signaling pathways [42]. Hsa_circ_0001461 is thought to regulate podocyte function as the upstream molecule of mir-30a.

However, there are some limitations in our work. First, our study is single-centered with a limited sample size. Larger independent cohorts are warranted to validate our findings. Second, the precise mechanism of hsa_circ_0001461 in the kidney has not been elucidated, and in-depth experiments are needed to reveal the function of circ. Finally, whether the differential circRNAs are specific to FSGS is uncertain, comparison among other renal diseases is desirable in future studies.

In summary, we have defined a distinctive circRNAs signature from kidney samples in children with FSGS. Renal circ_0001461 is identified as a potential biomarker for FSGS. This study provides a promising method to investigate the molecular pathogenesis of FSGS. Further investigations are warranted to reveal the functional mechanism of circRNAs in vivo and vitro.

Acknowledgements Thanks to Clinical Biological Resource Bank of Guangzhou Women and Children's Medical Center for providing all the clinical samples. We thank 
Yingyi He for her excellent suggestion for our manuscript. We extend our thanks to

\section{Editage (www.editage.cn) for English language editing.}

\section{Conflicts of interest The authors declare that they have no conflict of interest.}

\section{Reference}

1. Cattran DC, Rao P. Long-term outcome in children and adults with classic focal segmental glomerulosclerosis. Am J Kidney Dis 1998; 32: 72-79.

2. Savin VJ, Sharma R, Sharma M, et al. Circulating factor associated with increased glomerular permeability to albumin in recurrent focal segmental glomerulosclerosis. N Eng1 J Med 1996; 334: 878-883.

3. Garin EH, Mu W, Arthur JM, et al. Urinary CD80 is elevated in minimal change disease but not in focal segmental glomerulosclerosis. Kidney Int 2010;78:296302 .

4. Huang J, Liu G, Zhang YM, et al. Urinary soluble urokinase receptor levels are elevated and pathogenic in patients with primary focal segmental glomerulosclerosis. BMC Med 2014;12:81.

5. Cai X, Xia Z, Zhang C, et al. Serum microRNAs levels in primary focal segmental glomerulosclerosis. Pediatr Nephrol 2013; 28: 1797-1801.

6. Kristensen LS, Andersen MS, Stagsted LVW, et al. The biogenesis, biology and characterization of circular RNAs. Nat Rev Genet 2019; 20: 675-691.

7. Memczak S, Jens M, Elefsinioti A, et al. Circular RNAs are a large class of animal RNAs with regulatory potency. Nature 2013; 495: 333-338.

8. Jeck WR, Sorrentino JA, Wang K, et al. Circular RNAs are abundant, conserved, and associated with ALU repeats. RNA 2013; 19: 141-157.

9. Salzman J, Chen RE, 01sen MN, et al. Cell-type specific features of circular RNA expression. PLoS Genet 2013; 9: e1003777.

10. Maass PG, Glazar P, Memczak S, et al. A map of human circular RNAs in clinically relevant tissues. J Mol Med (Ber1) 2017; 95: 1179-1189.

11. Vo JN, Cieslik M, Zhang Y, et al. The landscape of circular RNA in Cancer. Cel1 2019; 176:869-881.

12. Aufiero S, Reckman YJ, Pinto YM, et al. Circular RNAs open a new chapter in cardiovascular biology. Nat Rev Cardiol 2019; 16: 503-514.

13. Zaiou M. CircRNAs signature as potential diagnostic and prognostic biomarker for diabetes mellitus and related cardiovascular complications. Cells 2020;9.

14. Hansen TB, Jensen TI, Clausen BH, et al. Natural RNA circles function as efficient microRNA sponges. Nature 2013; 495: 384-388.

15. Ashwal-Fluss R, Meyer M, Pamudurti NR, et al. CircRNA biogenesis competes with pre-mRNA splicing. Mol Cel1 2014; 56: 55-66.

16. Legnini I, Di Timoteo G, Rossi F, et al. Circ-ZNF609 Is a Circular RNA that Can Be Translated and Functions in Myogenesis. Mol Cel1 2017; 66: 22-37.

17. Pamudurti NR, Bartok 0, Jens M, et al. Translation of circRNAs. Mol Ce11 2017 ; 
66: 9-21.

18. Kong S, Tao M, Shen X, et al. Translatable circRNAs and 1ncRNAs: Driving mechanisms and functions of their translation products. Cancer Lett 2020; 483: $59-65$.

19. Luan J, Jiao C, Kong W, et al. CircHLA-C plays an important role in lupus nephritis by sponging miR-150. Mol Ther Nucleic Acids 2018; 10: 245-253.

20. Chen B, Li Y, Liu Y, Xu Z. CircLRP6 regulates high glucose-induced proliferation, oxidative stress, ECM accumulation, and inflammation in mesangial cells. J Cell Physiol 2019; 234: 21249-21259.

21. Hu W, Han Q, Zhao L, et al. Circular RNA circRNA_15698 aggravates the extracellular matrix of diabetic nephropathy mesangial cells via miR-185/TGFB 1. J Cell Physiol 2019; 234: 1469-1476.

22. Harvey SJ, Jarad G, Cunningham J, et al. Podocyte-specific deletion of dicer alters cytoskeletal dynamics and causes glomerular disease. J Am Soc Nephrol 2008; 19: 2150-2158.

23. Luo $\mathrm{Y}$, Wang $\mathrm{C}$, Chen $\mathrm{X}$, et al. Increased serum and urinary microRNAs in children with idiopathic nephrotic syndrome. Clin Chem 2013; 59: 658-666.

24. Zhang W, Zhang C, Chen H, et al. Evaluation of microRNAs miR-196a, miR-30a5P, and miR-490 as biomarkers of disease activity among patients with FSGS. Clin J Am Soc Nephrol 2014; 9: 1545-1552.

25. Wu J, Zheng C, Fan Y, et al. Downregulation of microRNA-30 facilitates podocyte injury and is prevented by glucocorticoids. J Am Soc Nephrol 2014; 25: 92-104.

26. Wang PL, Bao Y, Yee MC, et al. Circular RNA is expressed across the eukaryotic tree of life. PLoS One 2014; 9: e90859.

27. Chen L, Zhu D, Feng J, et a1. Overexpression of HHLA2 in human clear cell renal cell carcinoma is significantly associated with poor survival of the patients. Cancer Cell Int 2019; 19: 101.

28. Huang $\mathrm{Y}$, Zhang $\mathrm{Y}$, Jia L, et al. Circular RNA ABCB10 promotes tumor progression and correlates with pejorative prognosis in clear cell renal cell carcinoma. Int J Biol Markers 2019; 34: 176-183.

29. Kölling M, Seeger H, Haddad G, et al. The circular RNA ciRs-126 predicts survival in critically Ill patients with acute kidney injury. Kidney Int Rep 2018; 3: 1144-1152.

30. Jin X, Deng B, Ye K, et al. Comprehensive expression profiles and bioinformatics analysis reveal special circular RNA expression and potential predictability in the peripheral blood of humans with idiopathic membranous nephropathy. Mol Med Rep 2019; 20: 4125-4139.

31. Ma H, Xu Y, Zhang R, et al. Differential expression study of circular RNAs in exosomes from serum and urine in patients with idiopathic membranous nephropathy. Arch Med Sci 2019; 15: 738-753.

32. Ouyang Q, Huang Q, Jiang Z, et al. Using plasma circRNA_002453 as a nove1 biomarker in the diagnosis of lupus nephritis. Mol Immunol 2018; 101: 531538. 
33. Wei C, El Hindi S, Li J, et al. Circulating urokinase receptor as a cause of focal segmental glomerulosclerosis. Nat Med 2011; 17: 952-960.

34. Pereira LHM, da Silva CA, Monteiro M, et al. Podocin and uPAR are good biomarkers in cases of Focal and segmental glomerulosclerosis in pediatric renal biopsies. PLoS One 2019; 14: e0217569.

35. Gu H, Cheng X, Xu J, et al. Circular RNA circFAT1(e2) promotes osteosarcoma progression and metastasis by sponging miR-181b and regulating HK2 expression. Biomed Res Int 2020; 2020: 3589871.

36. Liu G, Huang K, Jie Z, et al. CircFAT1 sponges miR-375 to promote the expression of Yes-associated protein 1 in osteosarcoma cells. Mol Cancer 2018; $17: 170$.

37. Liu J, Li H, Wei C, et al. CircFAT1(e2) promotes papillary thyroid cancer proliferation, migration, and invasion via the miRNA-873/ZEB1 axis. Comput Math Methods Med 2020; 2020: 1459368.

38. Fang J, Hong $H$, Xue X, et al. A novel circular RNA, circFAT1(e2), inhibits gastric cancer progression by targeting miR-548g in the cytoplasm and interacting with YBX1 in the nucleus. Cancer Lett 2019; 442: 222-232.

39. Landgraf P, Rusu M, Sheridan R, et al. A mammalian microRNA expression atlas based on small RNA library sequencing. Cel1 2007; 129: 1401-1414.

40. Jiang L, Qiu W, Zhou Y, et al. A microRNA-30e/mitochondrial uncoupling protein 2 axis mediates TGF- $\beta$-induced tubular epithelial cell extracellular matrix production and kidney fibrosis. Kidney Int 2013; 84:285-296.

41. Wu J, Zheng $C$, Wang $X$, et al. MicroRNA-30 family members regulate calcium/calcineurin signaling in podocytes. J Clin Invest 2015; 125: 40914106.

42. Shi S, Yu L, Zhang T, et al. Smad2-dependent downregulation of miR-30 is required for TGF- $\beta$-induced apoptosis in podocytes. PLoS One 2013; 8: e75572. 
Table I. Primer pairs

\begin{tabular}{|c|c|}
\hline RNA transcript & Primer Pairs \\
\hline Hsa_circ_0001461 & $\begin{array}{l}\text { Fwd: CTCTGGCGTTGGTGTTTTCA } \\
\text { Rev: ACTGTCGGGAATCTGTCTCT }\end{array}$ \\
\hline Hsa_circ_0000605 & $\begin{array}{l}\text { Fwd:ACCTTTCTAACAGCCCAAGTAAA } \\
\text { Rev: TCTCCACTCAАCTCATCTGCT }\end{array}$ \\
\hline Hsa_circ_0001849 & $\begin{array}{l}\text { Fwd: CTCAAAGGGGCTTGGAAGAA } \\
\text { Rev: TGCCAGTTATCTTCAGTCCAGT }\end{array}$ \\
\hline Beta-actin, ActB & $\begin{array}{l}\text { Fwd: CCTCGCCTTTGCCGATCC } \\
\text { Rev: CTTCTGACCCATGCCCACC }\end{array}$ \\
\hline
\end{tabular}


Table II. Top 10 deregulated circRNAs in kidney tissues between FSGS patients and normal controls

\begin{tabular}{|c|c|c|c|}
\hline Up-regulated & Log2(Fold change) & Down-regulated & Log2(Fold change) \\
\hline circRNAs & FSGS vs. NC & circRNAs & FSGS vs. \\
\hline hsa_circ_0001461 & 3.613202 & hsa_circ_0000586 & -4.36883 \\
\hline hsa_circ_0000605 & 3.570701 & hsa_circ_0008838 & -4.336300 \\
\hline hsa_circ_0001849 & 3.329014 & hsa_circ_0001850 & -4.293221 \\
\hline hsa_circ_0006010 & 3.303928 & hsa_circ_0017348 & -3.862200 \\
\hline hsa_circ_0003261 & 2.992817 & hsa_circ_0044638 & -3.770316 \\
\hline hsa_circ_0007248 & 2.987162 & hsa_circ_0008659 & -3.737470 \\
\hline hsa_circ_0012460 & 2.8172672 & hsa_circ_0000475 & -3.588270 \\
\hline hsa_circ_0005523 & 2.7819287 & hsa_circ_0019985 & -3.498271 \\
\hline hsa_circ_0041267 & 2.7771908 & hsa_circ_0002470 & -3.327182 \\
\hline hsa_circ_0020670 & 2.7761928 & hsa_circ_0006706 & -3.309516 \\
\hline
\end{tabular}

FSGS, focal segmental glomerulosclerosis; NC, normal control 
Table III. The demographic and baseline characteristics of FSGS patients and NC

\begin{tabular}{|c|c|c|c|}
\hline Characteristic & FSGS & $\mathrm{NC}$ & $P$ value \\
\hline No. & 30 & 30 & \\
\hline Gender(M/F) & $22 / 8$ & $20 / 10$ & 0.573 \\
\hline Age of onset (year) & $3.31(2.00,3.48)$ & $3.29(1.95,4.50)$ & 0.806 \\
\hline $\operatorname{Serum}$ albumin $(\mathrm{g} / \mathrm{l})$ & $14.18 \pm 3.93$ & $35.12 \pm 4.67$ & $<0.001$ \\
\hline Serum creatinine $(\mu \mathrm{mol} / \mathrm{l})$ & $31.47 \pm 10.78$ & $30.33 \pm 12.18$ & 0.704 \\
\hline $\mathrm{eGFR}\left(\mathrm{ml} / \mathrm{min} / 1.73 \mathrm{~m}^{2}\right)$ & $138.04 \pm 24.41$ & $148.11 \pm 32.22$ & 0.178 \\
\hline $\begin{array}{l}\text { 24-hour } \\
\text { (mg/kg.d) }\end{array}$ & $340.39 \pm 145.07$ & - & - \\
\hline
\end{tabular}

FSGS, focal segmental glomerulosclerosis; NC, normal control; M, Male;

F, female; eGFR, estimated glomerular filtration rate 
Table IV. The demographic and baseline characteristics of patients with FSGS and MCD

\begin{tabular}{|c|c|c|c|}
\hline Characteristic & FSGS & MCD & $P$ value \\
\hline No. & 14 & 15 & \\
\hline Gender(M/F) & $12 / 2$ & $13 / 2$ & 1.00 \\
\hline Age of onset (year) & $3.15(1.90,6.05)$ & $4.00(2.80,5.50)$ & 0.512 \\
\hline Serum albumin $(g / 1)$ & $14.56 \pm 4.41$ & $13.75 \pm 4.32$ & 0.624 \\
\hline Serum creatinine $(\mu \mathrm{mol} / \mathrm{l})$ & $28.71 \pm 10.74$ & $30.80 \pm 14.09$ & 0.659 \\
\hline $\mathrm{eGFR}\left(\mathrm{ml} / \mathrm{min} / 1.73 \mathrm{~m}^{2}\right)$ & $156.72 \pm 31.61$ & $136.67 \pm 31.09$ & 0.097 \\
\hline $\begin{array}{l}\text { 24-hour } \\
\text { (mg/kg.d) }\end{array}$ & $197.71 \pm 121.80$ & $183.00 \pm 66.45$ & 0.687 \\
\hline
\end{tabular}

FSGS, focal segmental glomerulosclerosis; MCD, minimal change disease; $\mathrm{M}$, male; F, female; eGFR, estimated glomerular filtration rate 


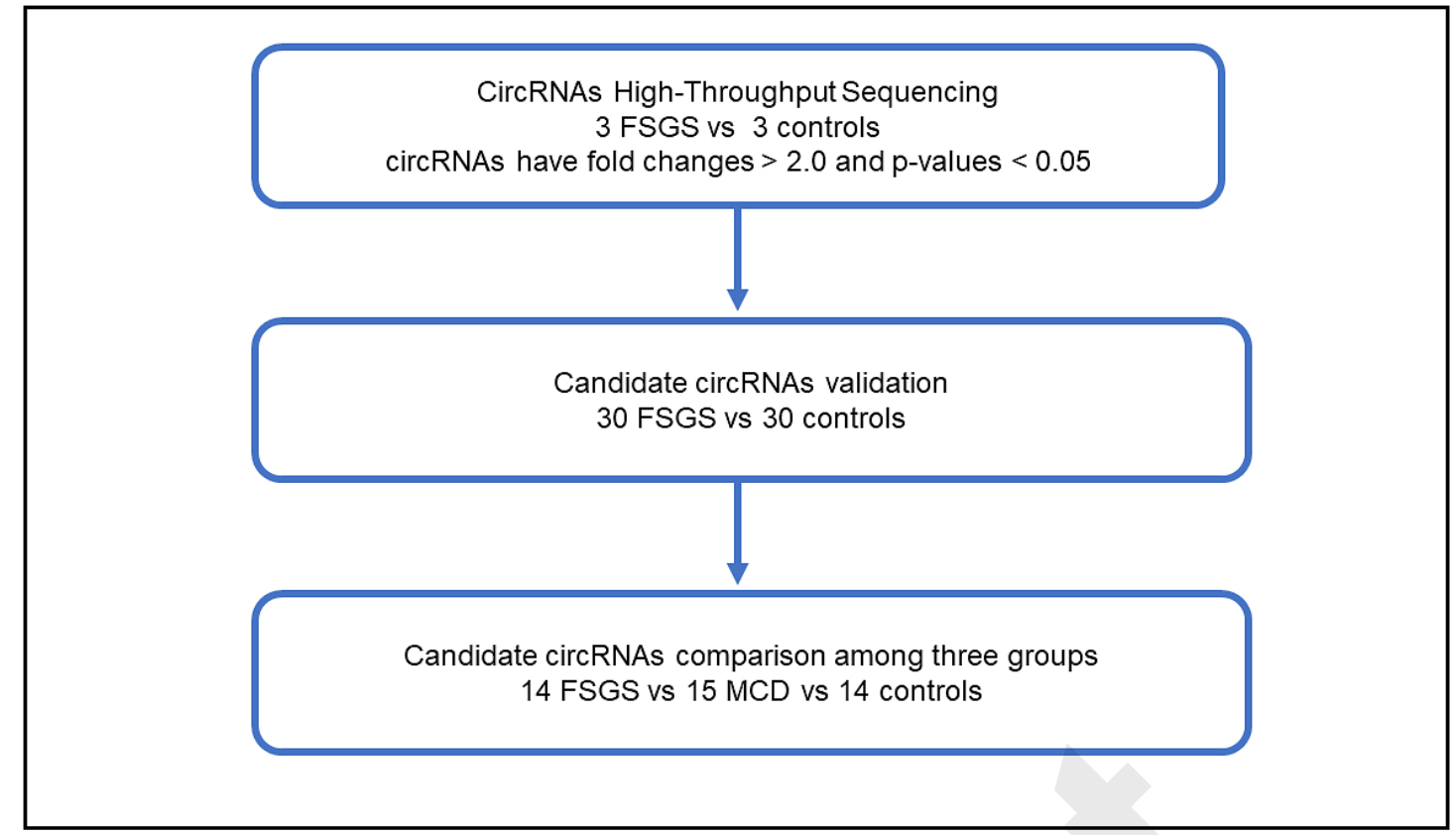

Figure 1. An overview of the design strategy. 

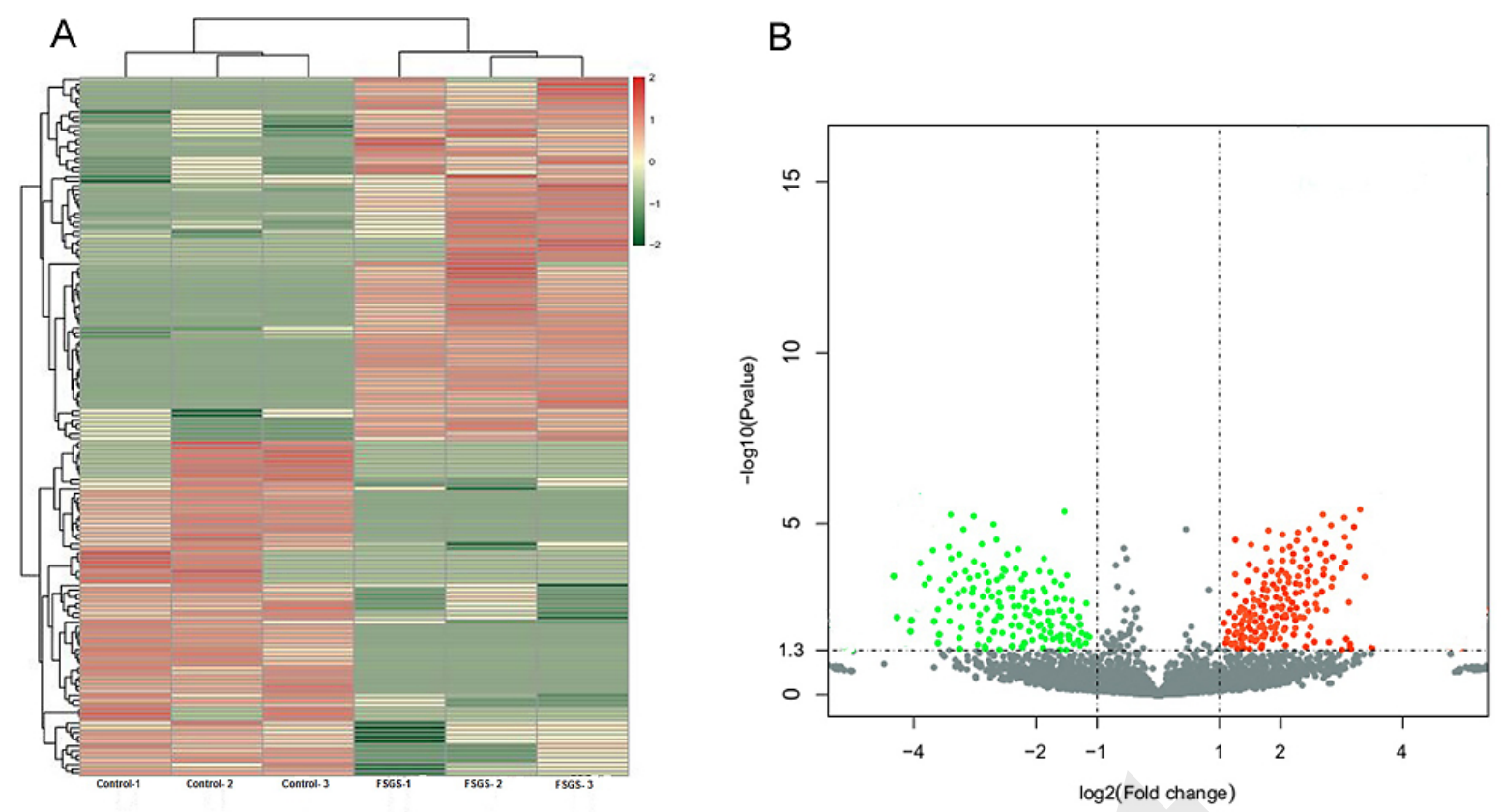

Figure 2. (A) The cluster heatmap shows the difference of circRNAs expression pattern among the samples. The color of red and green indicates high and low expression levels between two groups. (B) Volcano plot shows differentially expressed circRNAs in renal tissues of patients with FSGS and controls. FSGS, focal segmental glomerulosclerosis. 

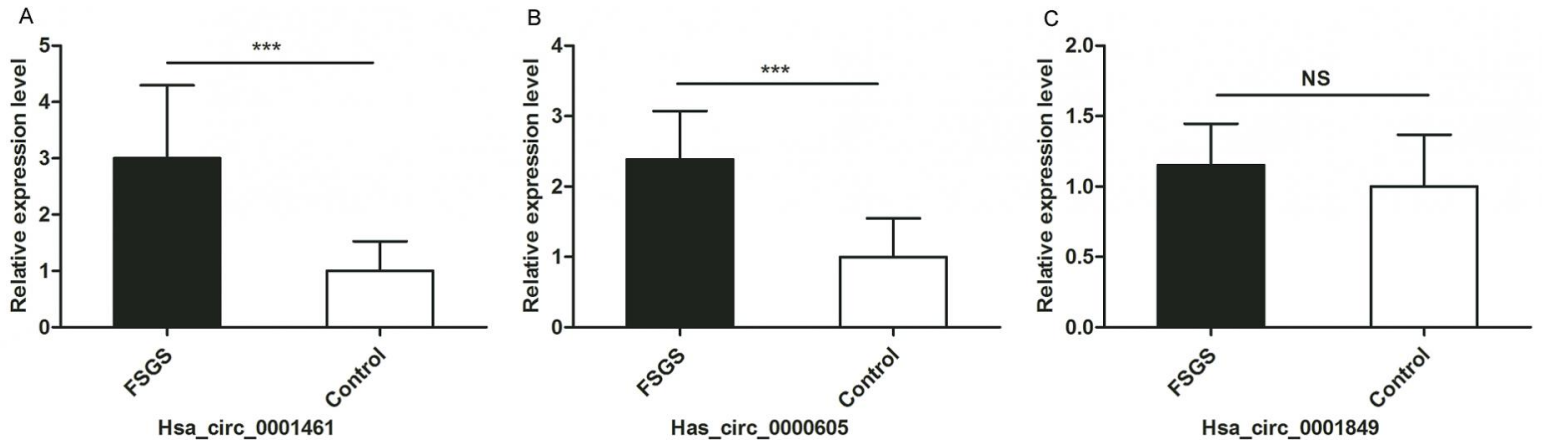

Figure 3. The expression levels of hsa_circ_0001461 (A), hsa_circ_0000605 (B) and has_circ_0001849 (C) in renal tissues of patients with FSGS compared to controls. ${ }^{* * *} \mathrm{P}<$ 0.001 ; NS, non-significant; FSGS, focal segmental glomerulosclerosis. 

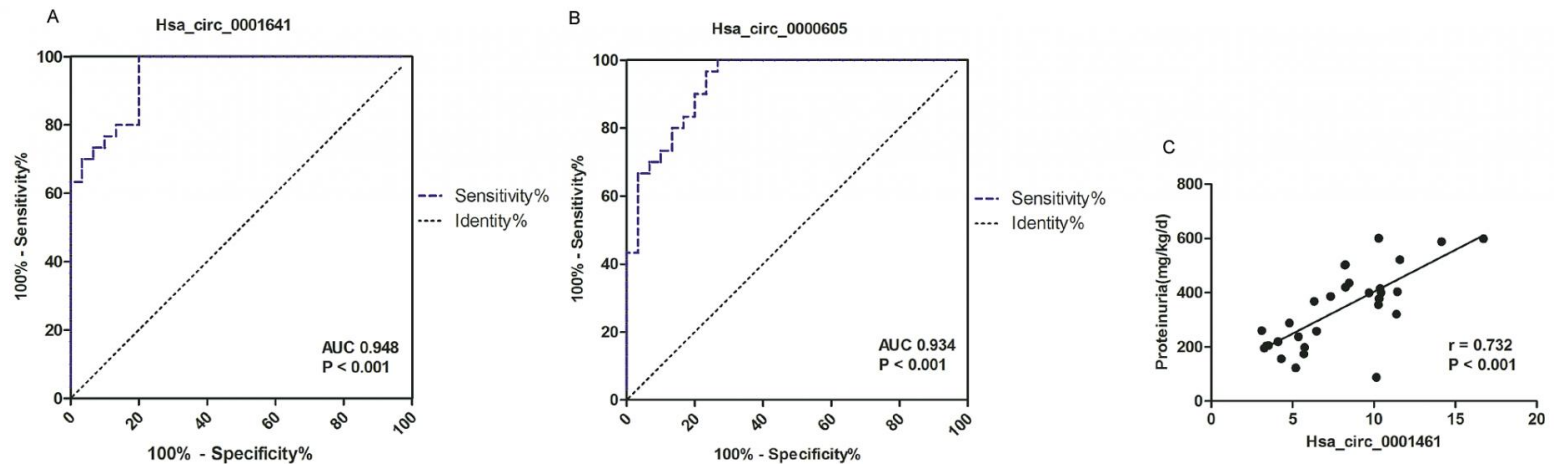

Figure 4. Receiver operating characteristic (ROC) analysis of patients with FSGS compared with controls regarding hsa_circ_0001461 (A) and hsa_circ_0000605 (B). (C)

Hsa_circ_0001461 was positively correlated with $24 \mathrm{~h}$ urinary protein in patients with FSGS $(r=0.732, p<0.001)$. FSGS, focal segmental glomerulosclerosis. 

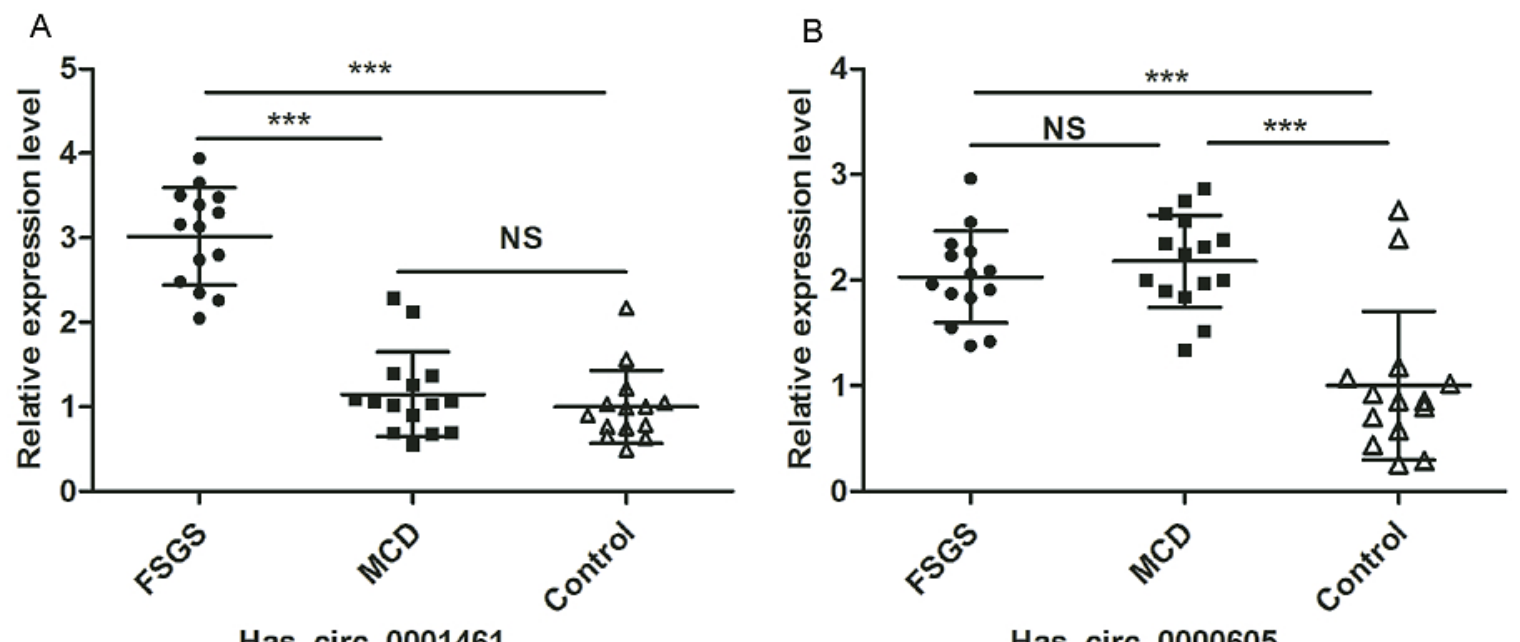

Figure 5. The expression levels of hsa_circ_0001461 (A) and hsa_circ_0000605 (B) in patients with FSGS compared with those with MCD and controls. ${ }^{* * *} \mathrm{P}<0.001$; NS, nonsignificant; FSGS, focal segmental glomerulosclerosis; MCD, minimal change disease. 
A

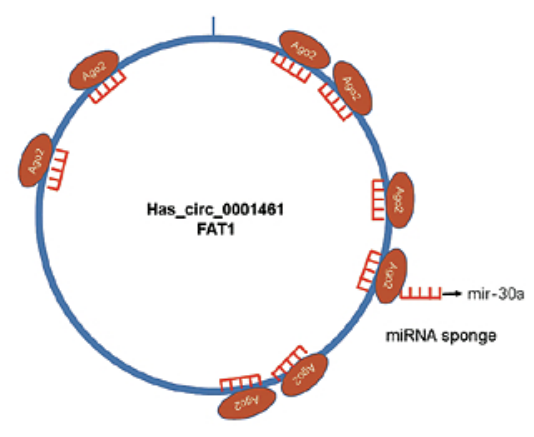

B

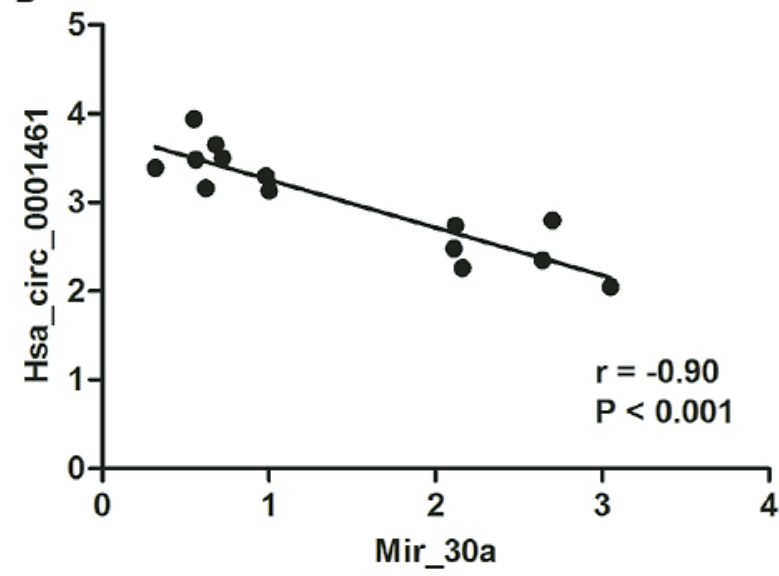

Figure 6. The interaction between renal hsa_circ_0001461 and mir-30 in FSGS. (A) Schematic cartoon of hsa_circ_0001461 in the pathogenesis of FSGS by sponging mir-30a. (B) Mir-30a was negatively correlated with the renal level of hsa_circ_0001461 $r=-0.90, p$ $<0.001)$. FSGS, focal segmental glomerulosclerosis. 